\title{
Clinical validation of the RTVue optical coherence tomography angiography image quality indicators
}

\author{
Noha Ali MMed(OphthSc) $)^{1,2}$ ｜ Danuta M. Sampson PhD ${ }^{1,3} \quad$ Alex Au Yong BSc ${ }^{\mathbf{1}}$ | \\ Rumaanah Jeewa BSc $^{1}$ | Saumya Rajgopal BSc ${ }^{1}$ | Deepaysh D. C. S. Dutt BSc ${ }^{1}$ | \\ Sharaf Mohamed MD $^{2}$ | Shehata Mohamed MD $^{2}$ | \\ Moreno Menghini MD FEBO ${ }^{4}$ | Alex Hansen MBBS $^{4}$ | \\ Fred K. Chen PhD FRANZCO', ${ }^{1,5}$

\footnotetext{
${ }^{1}$ Centre for Ophthalmology and Visual Science (Incorporating Lions Eye Institute), The University of Western Australia, Perth, Western Australia, Australia

${ }^{2}$ Department of Ophthalmology, Faculty of Medicine, Assiut University, Assiut, Egypt

${ }^{3}$ Surrey Biophotonics, Centre for Vision, Speech and Signal Processing and School of Biosciences and Medicine, The University of Surrey, Guildford, UK

${ }^{4}$ Department of Ophthalmology, Sir Charles Gairdner Hospital, Perth, Western Australia, Australia

${ }^{5}$ Department of Ophthalmology, Royal Perth Hospital, Perth, Western Australia, Australia
}

\section{Correspondence}

Dr Fred K. Chen, Lions Eye Institute, 2 Verdun Street, Nedlands, WA 6009, Australia.

Email: fredchen@lei.org.au

\begin{abstract}
Importance: All automated image quality indicators for en face optical coherence tomography angiography (OCTA) images require gold standard validation for determining optimum thresholds.

Background: A manual grading system (gold standard) for OCTA images was validated and compared to two automated image quality indicators: signal strength index (SSI) and scan quality index (SQI) generated by different software versions of the Optovue OCTA device.

Design: Retrospective cross-sectional study.

Participants: A total of 52 eyes of 52 healthy individual and 77 eyes of 51 patients with retinal vascular diseases.

Methods: A total of 129 OCTA images of the superficial vascular plexus were graded manually by three independent examiners. Each image was assigned grades 1 to 4 (1-2, unacceptable; 3-4, acceptable) masked to the softwaregenerated quality indicators.

Main Outcome Measures: Inter-grader agreement and comparison of the utility of SSI and SQI in discriminating between acceptable and unacceptable OCTA images.

Results: There was a substantial agreement between the three graders $(\kappa=0.63)$. Mean SSI and SQI was significantly different between acceptable
\end{abstract}


and unacceptable images $(P<.001)$. SQI outperformed SSI in separating acceptable from unacceptable images (areas under the receiver operating characteristic curve: 0.87 vs 0.80 ) and the optimum cut-off was $\geq 7$ for SQI and $\geq 70$ for SSI for acceptable images. Up to $30 \%$ of images with quality indicators reaching the optimum SQI and SSI cut-off thresholds still had unacceptable quality on manual grading. Unacceptable images were found in $33 \%$ and $66 \%$ of healthy and diseased eyes, respectively.

Conclusions and Relevance: SQI is closely related to manual grading but we caution reliance on the optimized threshold to determine image quality. SQI is superior to SSI in discriminating between acceptable and unacceptable images.

\section{K E Y W O R D S}

grading system, image quality, optical coherence tomography angiography, retinal angiography, retinal vascular disease

\section{1 | INTRODUCTION}

Optical coherence tomography angiography (OCTA) is gaining wide acceptance as a complementary, dye-free, clinical test, alongside fluorescein and indocyanine green angiography, in the investigation of retinal and choroidal vascular diseases. ${ }^{1}$ Clinically, OCTA has been used extensively to identify sub-clinical vascular changes in all forms of retinal and choroidal diseases including diabetic retinopathy (DR), ${ }^{2}$ retinal vein occlusion (RVO), ${ }^{3}$ inherited retinal diseases ${ }^{4}$ and choroidal neovascularization in agerelated macular degeneration (AMD). ${ }^{5}$ Currently, automated quantitative measurements of the capillary density and foveal avascular zone (FAZ) parameters can be generated to assess diseases progression and treatment response. ${ }^{6-8}$ However, the accuracy and reliability of these measurements are dependent on image quality. Therefore, image quality assessment is a critical aspect of initial OCTA evaluation.

Traditionally, the quality of a cross-sectional structural OCT scan is determined by the intensity of the backscattered OCT signal and reported in various terminology and scales according to the manufacturers. In contrast to structural OCT, signals in an OCTA image are generated by motion-contrast and then displayed as an en face image reconstructed from a series of densely packed raster scans. The signal strength index (SSI) (integer scale from 0 to 100), used by Optovue Inc. (Fremont, California) to quantify OCT scan quality, is defined as the average value of the supra-threshold logarithmicscale OCT reflectance signal with the threshold set just above the background noise. Although SSI does not take motion or projection artefact into consideration, previous studies have found an association between higher SSI and better image clarity, more accurate boundary segmentation and reduced variability of vessel density (VD) measurement ${ }^{9-11}$ while lower SSI was correlated with projection and banding artefacts, and greater variability of VR. ${ }^{12}$ In the most recent version of the AngioVue software (Optovue Inc., Fremont, California), an upgrade from Phase 6.5 to Phase 7.0, the SSI was replaced by a new parameter named scan quality index (SQI) (integer scale from 0 to 10), which combines SSI and an automated assessment of motion artefact and defocus measure. Previous studies have used a wide range of SSI thresholds (30-60) as the minimum requirement for inclusion in analysis. For SQI, a minimum of 6 or above has been recommended by Optovue as the threshold for acceptable image quality. However, the relationship between SSI and SQI has not been explored and the basis for manufacturer recommended minimum thresholds have not been tested against a clinical consensus (gold standard) grading system.

Therefore, the aims of this study are: (a) to describe and validate a simple 4-level manual grading system of en face OCTA image quality, (b) to investigate the agreement between the old and the new automated image quality indicators (SSI vs SQI), (c) to determine the optimum minimum SSI and SQI thresholds for acceptable OCTA image quality and (d) to explore factors that may predict unacceptable images.

\section{2 | METHODS}

This was a retrospective cross-sectional study of images from patients obtained at a single retina practice. The study protocol (RA/4/20/4275) was reviewed by the Human Ethics Office of Research Enterprise, the institutional review board (IRB) of the University of Western 
Australia and considered exempt from ethics review as this was a retrospective review of anonymized imaging data that was collected as part of routine clinical care. Images from a separate cohort of healthy subjects recruited prospectively under another study protocol (RA/4/1/8570) approved by the same IRB were also used in this study. The research was conducted in accordance to the tenets of the Declaration of Helsinki. All included subjects provided consent for their retinal images to be used for research.

\section{1 | Participants}

We reviewed our Optovue OCTA image database to identify patients with retinal vascular diseases (DR, RVO and Coats disease) irrespective of treatment history. Patients' medical charts were reviewed to confirm they did not have clinically significant lenticular opacities, corneal opacities or a history of refractive surgery or intraocular inflammation. DR grades were assigned R1, R2 and R3S (stable) as defined by the National Health Service diabetic eye screening programme. ${ }^{13}$ Healthy cohorts were recruited prospectively from volunteers and patients attending the eye clinic with no history of ocular disease and normal retinal examination and imaging. All healthy subjects had a best-corrected ETDRS letter score of 85 or greater. All retinal patients and healthy subjects underwent retinal imaging after pupils were dilated using $1 \%$ tropicamide with or without $2.5 \%$ phenylephrine eye drops. Those with any macular pathology on structural OCT scan were excluded from the prospective healthy control cohort. One eye was included from the healthy and the diseased cohort except in cases of bilateral retinal pathology where both eyes were included.

\section{2 | OCTA protocol}

OCTA images were acquired using the RTVue XR Avanti system (version 2016.1.0.26, Optovue, Inc., California) prior to software upgrade from Phase 6.5 to Phase 7.0. The instrument operates at a central wavelength of $840 \mathrm{~nm}$ with a bandwidth of $45 \mathrm{~nm}$ and at a speed of 70000 A-scans per second. The system provides an axial resolution of $5 \mu \mathrm{m}$ in tissue and the diameter of the beam spot at the retinal plane is around $22 \mu \mathrm{m}$. The $3 \times 3 \mathrm{~mm}$ OCTA scans collected in this study consists of 304 densely packed B-scans and each B-scan is derived from 304 Ascans with 640 pixels along the length of A-scan. At a speed of 209-line scans per second, each volume scan (304 lines $\times 2$ B-scans per line) is acquired within 3 seconds. Two consecutive volume scans were acquired at orthogonal direction ( $x$-fast and $y$-fast) and they were aligned and merged automatically to reduce motion artefacts. ${ }^{14}$ Flow signals are derived from split-spectrum amplitude decorrelation angiography algorithm so that angiographic-like images is generated. ${ }^{15}$ SSI and SQI were collected from all eligible patients before and after software upgrade from Phase 6.5 to Phase 7.0, respectively. Eyes were split into six groups by SSI values of: $\leq 50,51-60,61-70,71-80$ and $81-90$ or into five groups by SQI value into: $\leq 5,6,7,8$ and 9 .

\section{3 | Manual image quality grading}

OCTA images of the superficial vascular plexus, generated from the Phase 6.5 version software, were graded for (a) the clarity of the vascular structures and (b) the presence of residual image artefacts by three independent trained graders (F.K.C., D.M.S., N.A.) using a new grading system (Figure 1 and Table 1). Each grader was given a short tutorial on the grading system reinforced by the availability of illustrative cases for each of the four categories. These cases were not included in this study cohort. Grading was performed independently and masked to the results of the other graders and to the automated grading scores (SSI or SQI). Grades 1 and 2 images are considered as unacceptable for quantitative analysis while grades 3 and 4 are considered as acceptable. In cases where there were disagreement, a "final" grade was assigned based on consensus after real-time discussion between all three graders.

\subsection{Statistical analysis}

Patient demographics were summarized by mean and SD. Inter-grader agreement of the manual grading between the three graders was quantified by Fleiss kappa coefficient $(\kappa)$. The correlation between SSI and SQI was examined by using the Kruskal-Wallis test to determine if there were statistically significant differences in the SSI across the five SQI categories. Dunn's post hoc tests with Bonferroni correction was performed, if there was a significant difference.

Next, we investigated whether SSI or SQI correlated with manual grading by using the Kruskal-Wallis test to determine if SSI or SQI differed across the four manual grades. The ability of SSI and SQI to discriminate acceptable (grades 3 and 4) from unacceptable (grades 1 and 2) images were further analysed by Mann-Whitney test to determine if SSI and SQI were significantly different between these two groups of images. The proportions of acceptable and unacceptable images in each category of 
(A)

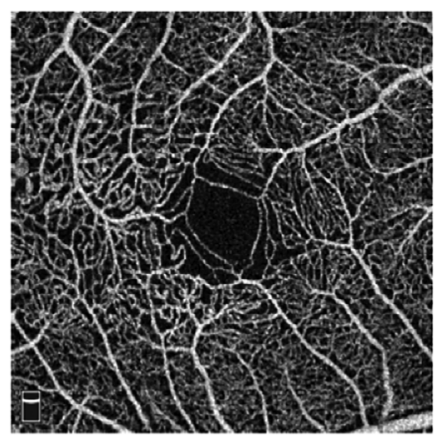

(D)

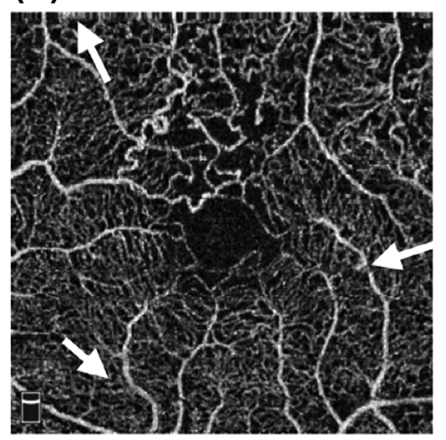

(G)

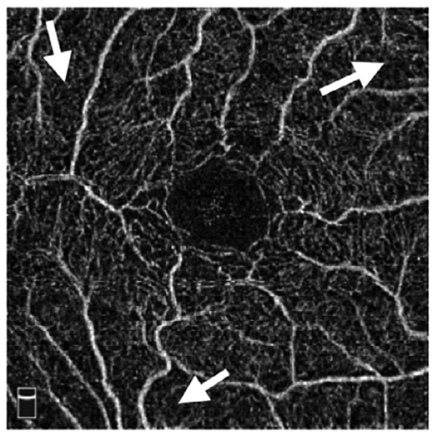

(J)

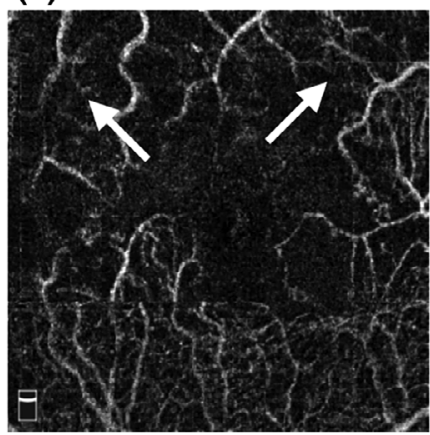

(B)

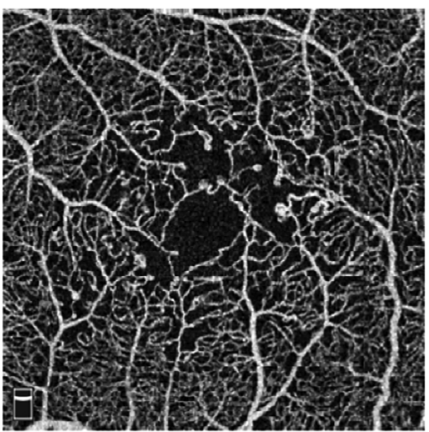

(E)

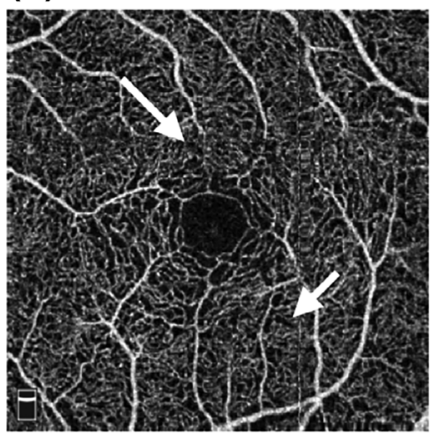

(H)

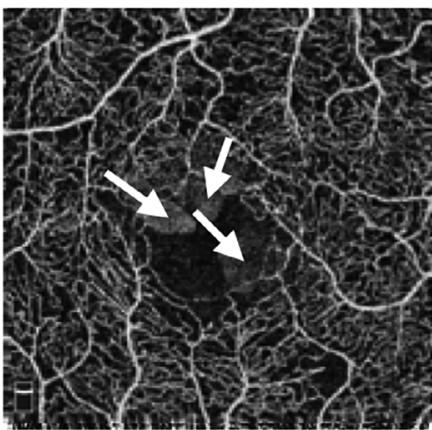

(K)

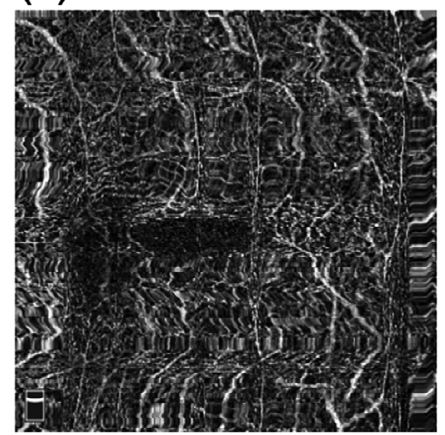

(C)

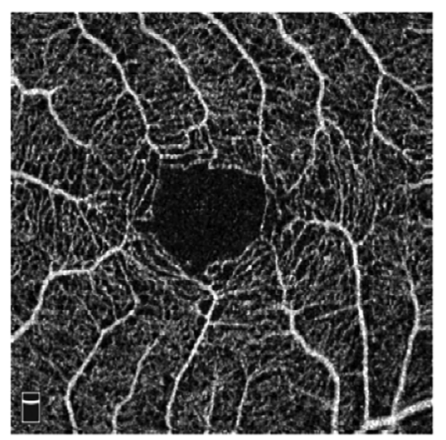

(F)

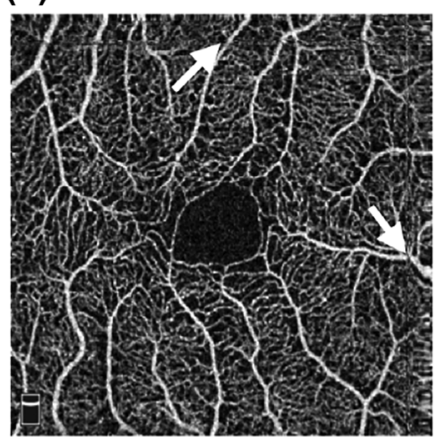

(I)

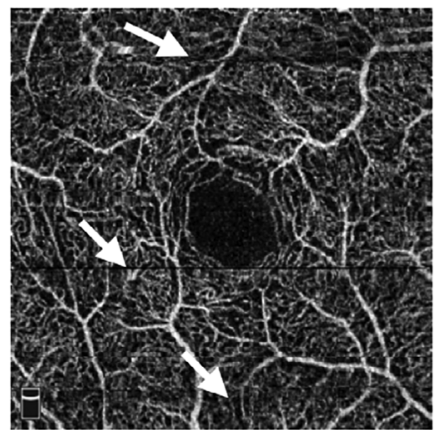

(L)

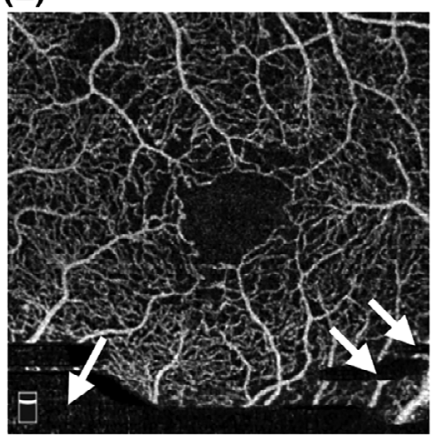

F I G U R E 1 Grades of the manual grading system. Grade 4: clear continuous vessels without motion or false motion signals (A-C). Grade 3: subtle lines of motion artefacts (white arrows, D-F). Grade 2: vascular structures discontinuous or attenuated (white arrows, G), false motion signals (white arrow, $\mathrm{H}$ ), thick bands of motion artefacts (white arrows, I). Grade 1: No clear vascular structures or marked discontinuity of the vascular tree, bands of motion artefacts (bright and dark band), missing areas of the flow signal (white arrows, J-L)

SQI and SSI values were tabulated. Receiver operating characteristic (ROC) curves were used to determine the optimum cut-off threshold in SSI and SQI for separating manually graded acceptable (grades 3 and 4) from unacceptable (grades 1 and 2) images and areas under the ROC curves (AUC) were calculated. 
Logistic regression was performed to ascertain the effects of age (years), sex (male vs female), visual acuity (ETDRS letter score), retinal pathology (present or absent) and macula oedema (present or absent) on the likelihood of poor image quality (grades 1 and 2). One

TA B L E 1 Definition of the manual grading system

\begin{tabular}{|c|c|}
\hline Grade & Definition \\
\hline $\begin{array}{l}\text { Grade 4: Excellent image } \\
\text { quality }\end{array}$ & $\begin{array}{l}\text { The vascular structures are } \\
\text { continuous } \\
\text { The vessels are clearly seen } \\
\text { No lines of motion artefact } \\
\text { No false motion signal }\end{array}$ \\
\hline Grade 3: Good image quality & $\begin{array}{l}\text { The vascular structures are } \\
\text { continuous } \\
\text { The vessels are clearly seen } \\
\text { Subtle lines of motion } \\
\text { artefacts } \\
\text { No false motion signal }\end{array}$ \\
\hline $\begin{array}{l}\text { Grade 2: Poor image quality } \\
\text { (any one of these features) }\end{array}$ & $\begin{array}{l}\text { Vascular structures are } \\
\text { visible but discontinuous } \\
\text { Blurry vascular margin } \\
\text { Bands of motion artefacts } \\
\text { (bright or dark) } \\
\text { Clearly false motion signal }\end{array}$ \\
\hline $\begin{array}{l}\text { Grade 1: Degraded image } \\
\text { quality }\end{array}$ & $\begin{array}{l}\text { Vascular structures are } \\
\text { hardly visible or distorted } \\
\text { Areas of the fundus image } \\
\text { missing } \\
\text { Bands of motion artefacts } \\
\text { (bright or dark) } \\
\text { Clearly false motion signal }\end{array}$ \\
\hline
\end{tabular}

eye of each patient was used in this analysis to avoid inter-ocular correlation. Odds ratio (OR) and 95\% confidence intervals (CI) were reported. Statistical analysis was carried out using SPSS software (version 24.0, SPSS Inc., Chicago, Illinois). A $P$-value of less than .05 was considered statistically significant.

\section{3 | RESULTS}

\section{1 | Patient characteristics}

A total of 129 OCTA images from 52 eyes of 52 healthy subjects and 77 eyes of 51 patients with retinal vascular disease were selected for inclusion in the analysis. These images were exported through the older, Phase 6.5 version, software for manual grading and their SSI (older software version) and SQI (newer software version) were recorded. Demographic and clinical diagnoses are shown in Table 2. Among the 51 patients with retinal disease, 29 had DR, 13 had RVO and 9 had adult Coats disease.

\section{2 | Inter-grader agreement}

There was a substantial agreement between the three graders $(\kappa=0.63$, Tables S1-S3). Overall, $74.5 \%$ of the manual image quality grading had complete agreement between the three graders while $24 \%$ had a disagreement between the graders by one-step level and only $1.5 \%$ had a disagreement by two-step levels of the quality grading. Following the real-time discussion between graders in cases of grading disagreement, 23 (17.8\%), 45 (34.9\%), $51(39.5 \%)$ and $10(7.8 \%)$ images were assigned the final grades of 1, 2, 3 and 4, respectively.

T A B L E 2 Demographic characteristic of eligible subjects

\begin{tabular}{|llllll|}
\hline Subjects & $\begin{array}{l}\text { No. of eyes } \\
\text { included }\end{array}$ & $\begin{array}{l}\text { No. of } \\
\text { patients }\end{array}$ & $\begin{array}{l}\text { Age, } \\
\text { mean (SD) }\end{array}$ & $\begin{array}{l}\text { Sex } \\
\text { M:F ratio }\end{array}$ & $\begin{array}{l}\text { VA in ETDRS letter score, } \\
\text { mean (SD) [range] }\end{array}$ \\
\hline Healthy controls & 52 & 52 & $37(14)$ & $24: 28$ & $85^{\text {a }}$ \\
\hline Retinal patients & 77 & 51 & $55(16)$ & $27: 24$ & $75(11)[42-90]$ \\
\hline DR, R1 & 23 & 12 & $60(13)$ & $8: 4$ & $80(6)[65-90]$ \\
\hline DR, R2 & 9 & 5 & $62(8)$ & $2: 3$ & $72(13)[50-87]$ \\
\hline DR, R3S & 23 & 12 & $48(15)$ & $6: 6$ & $72(9)[45-82]$ \\
\hline RVO & 13 & 13 & $64(14)$ & $3: 10$ & $73(16)[42-87]$ \\
\hline ACD & 9 & 9 & $42(20)$ & $8: 1$ & $77(12)[57-89]$ \\
\hline Entire cohort & 129 & 103 & $48(18)$ & $51: 52$ & $79(8)[42-90]$ \\
\hline
\end{tabular}

Abbreviations: ACD, adult Coats disease; DR, diabetic retinopathy; ETDRS, Early Treatment Diabetic Retinopathy Study; F, female; M, male; R1, mild to moderate non-proliferative diabetic retinopathy; R2, severe non-proliferative diabetic retinopathy; R3S, stable treated proliferative diabetic retinopathy; RVO, retinal vein occlusion; VA, visual acuity.

${ }^{\mathrm{a}}$ Visual acuity for the healthy controls is 85 ETDRS letters or above. 


\subsection{Inter-indices comparison}

There is a trend for greater SSI values in images with increasing SQI (Figure 2A). Those with SQI of 8 or 9 had a significantly higher SSI compared to those with SQI of $\leq 5,6$ or $7(P<.001)$. There were no significant differences in SSI between SQI of $\leq 5,6$ or 7 ( $P=.132$ to 1.0$)$ or between SQI of 8 and 9 ( $P=.619$ to 1.0 , Table 3$)$. However, the range of SSI values overlapped considerably between SQI groups. For example, the range of SSI (39-85) from images with SQI $\leq 5$ overlapped with range of SSI (73-88) from images with SQI $=9$ (Table 4).

\section{4 | Optimum thresholds in automated indices for acceptable images}

Both, SSI and SQI increased with improving manually graded image quality (from grades 1 to 4 , Kruskal-Wallis test, $P<.001$, Table 5). However, pairwise testing showed that SQI was the only metric that showed significant difference between grades 1 and $2(P=.003)$. Neither SQI nor SSI values were significantly different between grades 3 and 4 as illustrated in Figure 2B,C. The ability of SSI and SQI to discriminate between acceptable (grades 3 and 4 ) and unacceptable (grades 1 and 2) images were further examined.

Mann-Whitney $U$ test demonstrated a significant difference in overall SSI and of SQI $(P<.001)$ between manually graded acceptable (grades 3 and 4 ) and unaccepted (grades 1 and 2) images. The percentage of acceptable images (grades 3 and 4 ) increased from $23 \%$ in the SSI $=51-60$ group to $81 \%$ in the SSI $=81-90$ group (Table 6). If only images with SSI $>70$ were kept for analysis, $29 \%$ (17 of 58) would still be graded as unacceptable quality. Similarly, proportion of acceptable images increased from $47 \%$ in the SQI $=7$ group to $89 \%$ in the SQI $=9$ group (Table 7). If only images with SQI $\geq 7$ were kept for analysis, $32 \%$ (29 of 90) would still be graded as unacceptable quality. All images with SSI of $\leq 50$ or SQI of 6 or less were graded as unacceptable (grades 1 and 2) images. Figure 3 shows the ROC for both SSI and SQI for discriminating between unacceptable and acceptable images. We found that SQI has a better discrimination $(\mathrm{AUC}=0.87)$ than SSI (AUC $=0.80$ ), with a cut-off threshold of between 6 and 7 for SQI and between 69 and 70 for SSI providing the maximum sensitivity and specificity to discriminate unacceptable from the acceptable quality OCTA images.

\section{5 | Predictors of poor quality OCTA images}

Although unacceptable quality OCTA images (grades 1 and 2) were found twice as likely in eyes with retinal
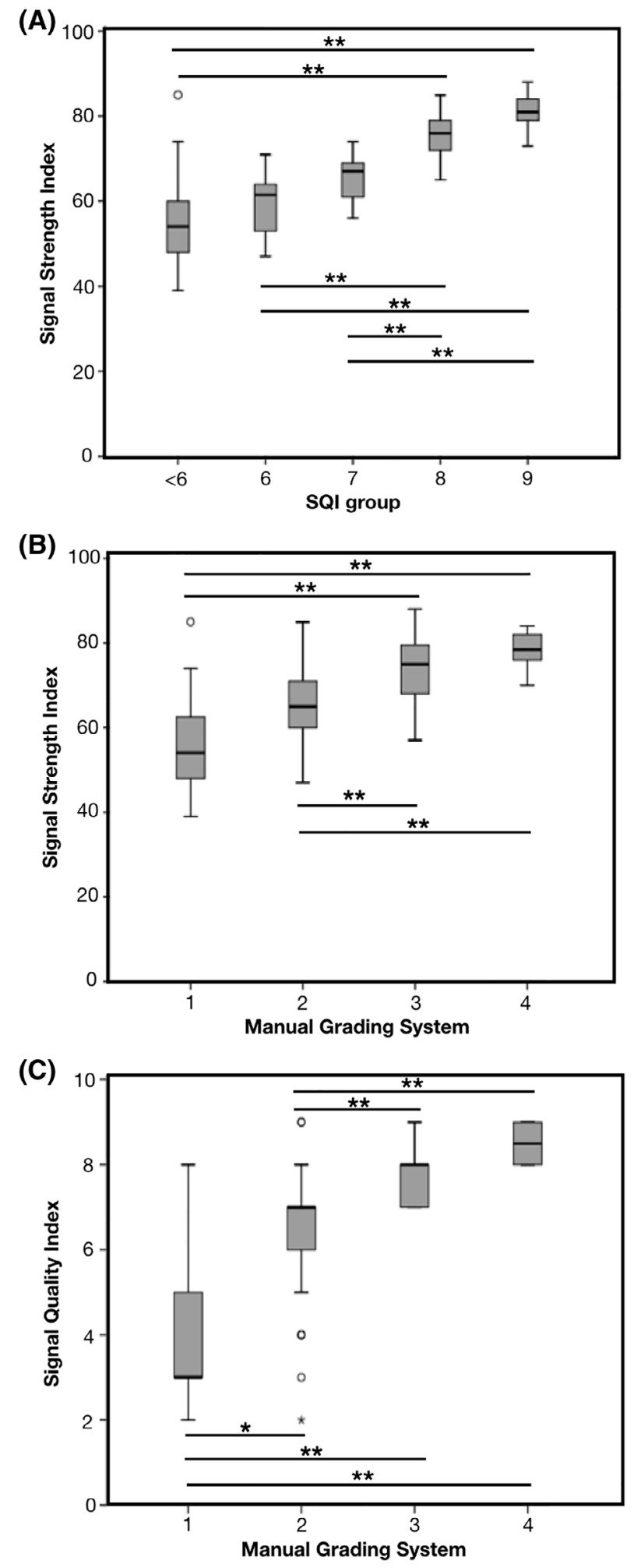

F I G U RE 2 Boxplots showing the range of signal strength index for each scan quality index group ( $\leq 5,6,7,8$ and $9, A)$, scan quality index for the four levels of consensus manual grades (B), and signal strength index for the four levels of consensus manual grades (C). Kruskal-Wallis tests were performed for each system. Differences between pairs identified by post-hoc DunnBonferroni tests are represented in the figure $(* P<.05 ; * * P<.001)$ 
T A B LE 3 Pair-wise comparison in signal strength index between different scan quality index groups
T A B L E 4 Distribution in signal strength index across scan quality index values

\begin{tabular}{llllll} 
& SQI & & & \\
\cline { 2 - 6 } Adjusted $\boldsymbol{P}$-values SQI & $\mathbf{5}$ or less & $\mathbf{6}$ & $\mathbf{7}$ & $\mathbf{8}$ & $\mathbf{9}$ \\
$\leq 5$ & 1.0 & 1.0 & .132 & $<.001$ & $<.001$ \\
6 & - & 1.0 & 1.0 & $<.001$ & $<.001$ \\
7 & - & - & 1.0 & $<.001$ & $<.001$ \\
8 & - & - & - & 1.0 & .619 \\
9 & - & - & - & - & 1.0 \\
\hline
\end{tabular}

Notes: Shaded: statistically significant; $P$-values are Bonferroni adjusted. Abbreviations: SQI, scan quality index.

\begin{tabular}{lccccc} 
& SQI & & & & \\
\cline { 2 - 6 } SSI & $\mathbf{5}$ or less & $\mathbf{6}$ & $\mathbf{7}$ & $\mathbf{8}$ & $\mathbf{9}$ \\
Median (range) & $54(39-85)$ & $62(47-71)$ & $67(56-74)$ & $76(65-85)$ & $81(73-88)$ \\
Mean (SD) & $55.2(10.8)$ & $59.5(6.9)$ & $65.3(5.1)$ & $75.8(4.3)$ & $81.1(3.6)$ \\
\hline
\end{tabular}

Abbreviations: SQI, scan quality index; SSI, signal strength index.

\begin{tabular}{|c|c|c|c|c|}
\hline \multirow[b]{2}{*}{ Image grade } & \multicolumn{2}{|c|}{ Unacceptable } & \multicolumn{2}{|c|}{ Acceptable } \\
\hline & Grade 1 & Grade 2 & Grade 3 & Grade 4 \\
\hline \multicolumn{5}{|l|}{ SSI } \\
\hline Mean (SD) & $57(12)$ & $65(9)$ & $73(8)$ & $78(4)$ \\
\hline Median (range) & $54(39-85)$ & $65(47-85)$ & 75 (57-88) & $79(70-84)$ \\
\hline \multicolumn{5}{|l|}{ SQI } \\
\hline Mean (SD) & $3.8(1.7)$ & $6.4(1.4)$ & $7.8(0.7)$ & $8.5(0.5)$ \\
\hline Median (range) & $3(2-8)$ & $7(2-9)$ & $8(7-9)$ & $8.5(8-9)$ \\
\hline
\end{tabular}

T A B L E 5 Average signal strength index (SSI) and scan quality index (SQI) for each grade

T A B L E 6 Distribution of acceptable and unacceptable image among different signal strength index groups

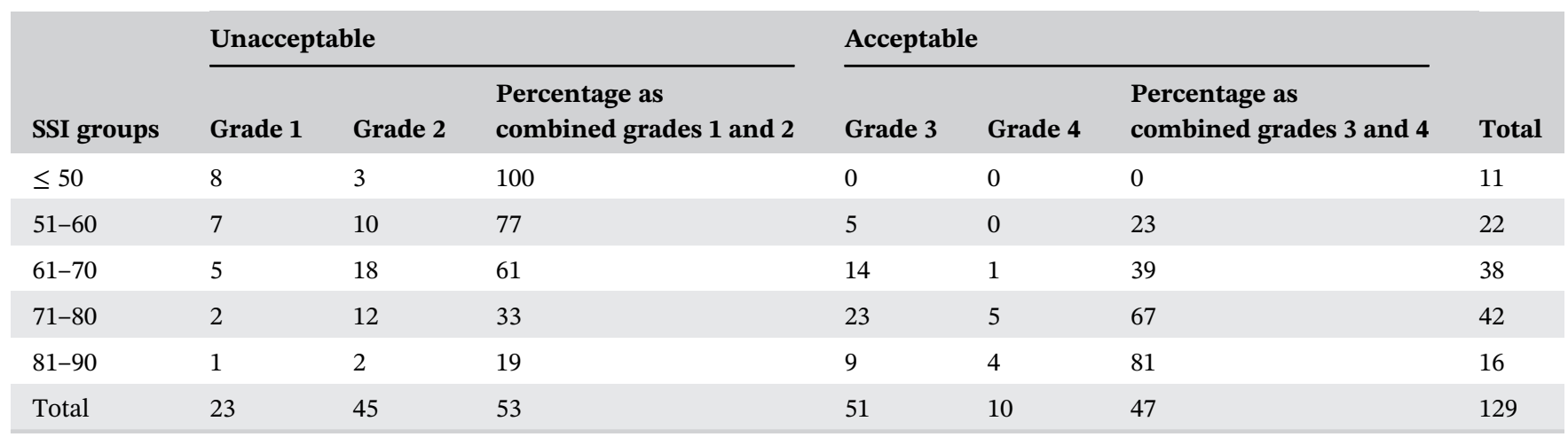

Abbreviation: SSI, signal strength index.

disease compared to healthy subjects (66\% vs $33 \%$, $P<.001$ ), images from eyes with macular atrophy had a particularly high frequency of unacceptable quality (88\%). The distribution of the accepted and unaccepted images according to the various diagnoses and the maculopathy status are shown in Tables 8 and 9. To control for confounding patient and ocular factors, a binary logistic regression was performed to ascertain the effects of age, gender, visual acuity, retinal pathology and macula oedema on the likelihood of obtaining an unacceptable OCTA image quality (grades 1 and 2). We included one eye from each subject $(N=103)$ in this analysis. The right and left eyes of the 26 subjects that had images from both eyes available were sampled in tests 1 and 2, respectively. 
T A B L E 7 Distribution of acceptable and unacceptable image amongst different scan quality index groups

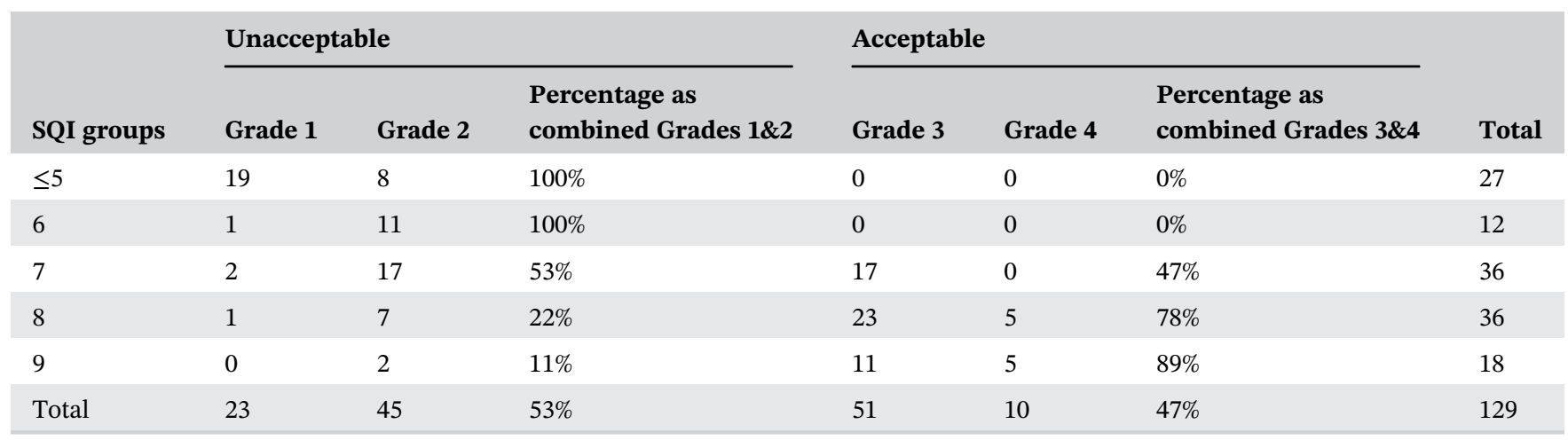

Abbreviation: SQI, scan quality index.
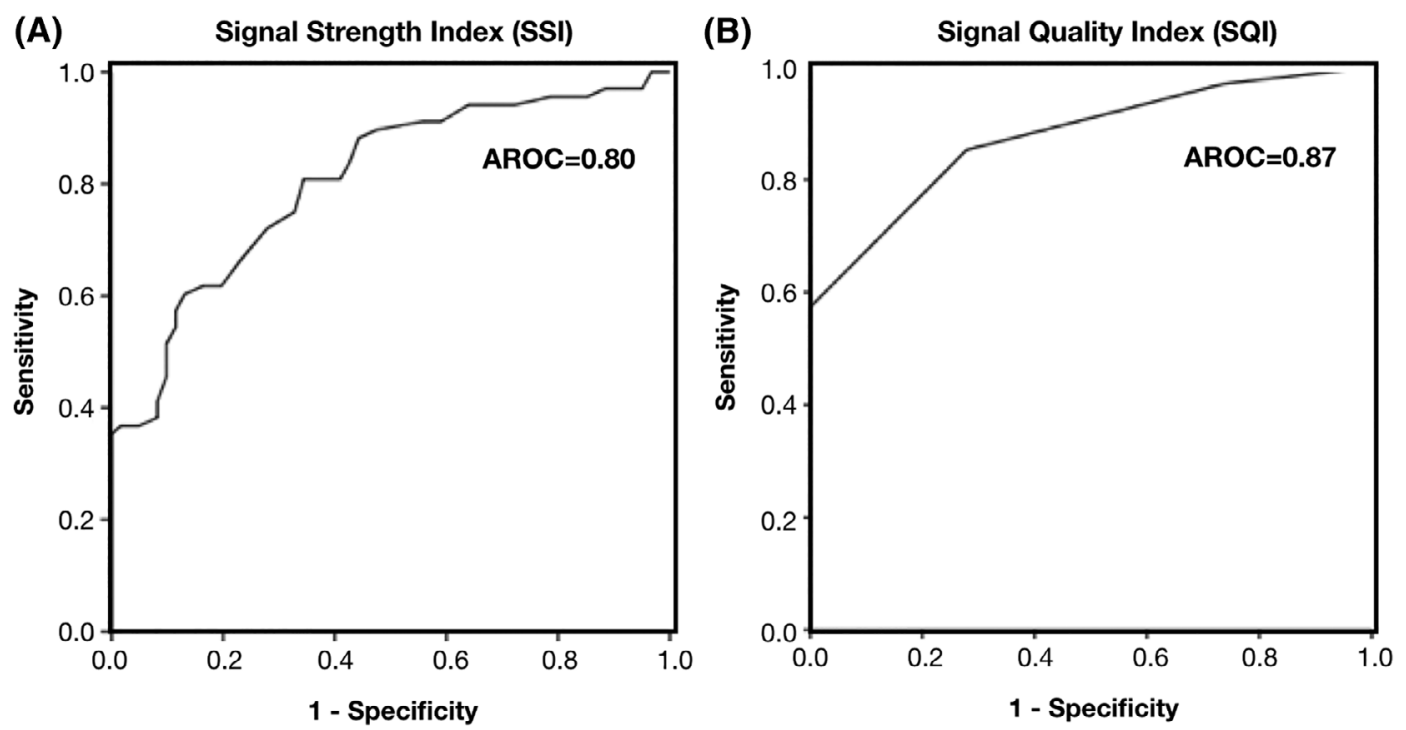

F I G U R E 3 Receiver operating characteristic curves showing abilities of signal strength index (A) and scan quality index (B) in discriminating unacceptable (grade 1 and 2) from acceptable (grade 3 and 4) images. Area under the curves (AUC) is greater for the newer signal quality index (SQI) than the signal strength index (SSI) derived from the Phase 6.5 version software

The logistic regression model was statistically significant (test 1: $\chi^{2}(5)=14.263, P=.014$ and test $2: \chi^{2}(5)=21.438$, $P=.001)$. These models explained $17.3 \%$ and $25.1 \%$ (Nagelkerke $R^{2}$ ) of the variance in image quality and correctly classified $66.0 \%$ and $69.9 \%$ of the cases, respectively. Decreasing ETDRS letter score was associated with an increasing likelihood of poor image quality (test 1 : $\beta=-.068, \mathrm{OR}=0.934, \mathrm{CI}: 0.871-1.002, P=.056$ and test 2 : $\beta=-.077, \mathrm{OR}=0.926, \mathrm{CI}: 0.859-0.998, P=0.044)$.

\section{4 | DISCUSSION}

We reported substantial inter-grader agreement in our simple four-level manual grading system of OCTA image quality that incorporates assessment of vessel continuity and motion artefact. In contrast, there was significant discrepancy between the two automated grading, SSI and SQI. Using adjudicated manual grading as the gold standard, we showed that an SSI threshold of $\geq 70$ or an SQI threshold of $\geq 7$ as the optimum cut-off for acceptable image quality with maximum sensitivity and specificity. However, up to $30 \%$ of images with SSI or SQI reaching these cut-off thresholds will still have unacceptable quality based on manual grading system. We found decreased visual acuity as the main predictor of unacceptable image quality after controlling for patient and ocular factors such as type of disease and maculopathy status.

\section{1 | Manual grading indices}

Several studies have described an image quality grading system for OCTA. Munk et $\mathrm{al}^{16}$ reported a subjective 
T A B L E 8 Distribution of manual grading stratified by clinical diagnosis or maculopathy type

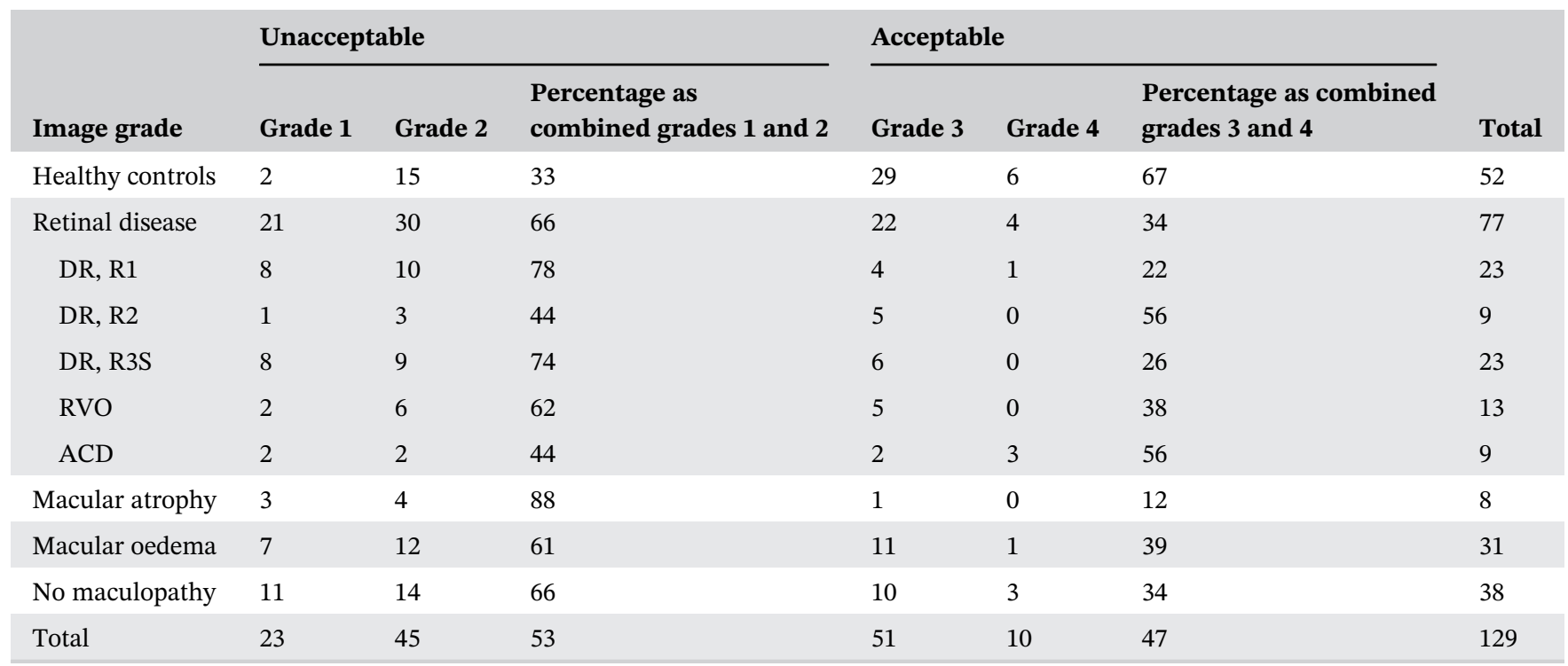

Abbreviations: ACD, adult Coat disease; DR, diabetic retinopathy; R1, mild to moderate non-proliferative diabetic retinopathy; R2, severe non-proliferative diabetic retinopathy; R3S, stable treated proliferative diabetic retinopathy; RVO, retinal vein occlusion.

T A B L E 9 Distribution of the grades stratified by presence of maculopathy

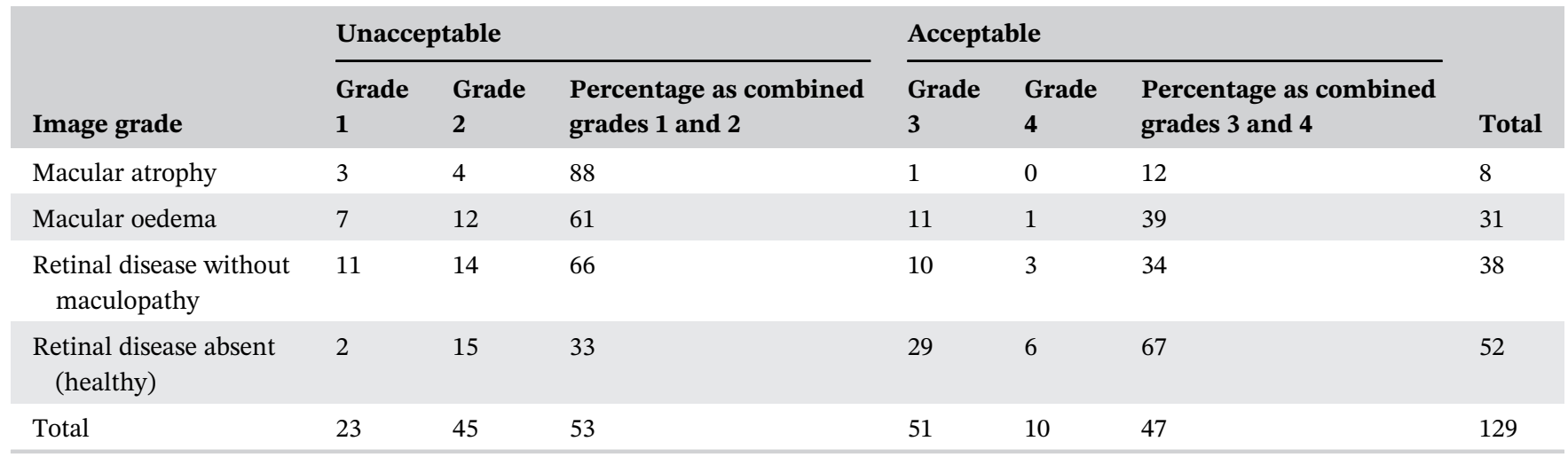

grading system to compare the image quality between four commercially available OCTA devices. Lauermann et $\mathrm{al}^{17}$ also recently described a motion artefacts score (MAS) and segmentation accuracy score (SAS) to compare the frequency of motion artefacts and segmentation errors between healthy subjects and patients with retinal diseases. Cardoso et $\mathrm{al}^{18}$ graded the OCTA images into adequate, fair and poor depending on the degree of confidence in reading the photographic features as a part of their study to evaluate the utility of OCTA in RVO. However, none of these studies provide a quality protocol to determine which images are acceptable in the clinical practice for quantitative analysis. We demonstrated that our grading system has a good inter-observer agreement $(\kappa=0.63)$ and the four-step level is easy to use for determining acceptability of OCTA image for quantitative analysis. Our inter-observer agreement is similar to that reported by Munk et al. ${ }^{16}(\kappa=0.52)$ for superficial capillary plexus on the Optovue device. Lauermann et $\mathrm{al}^{17}$ reported $89 \%$ agreement in their MAS and SAS grading between two graders, whereas we had only $74.5 \%$ agreement between the three graders and 67\%-83\% agreement between two graders (Tables S1-S3).

\section{2 | Automated grading indices}

SSI and SQI are automated quality indices provided by the Phase 6.5 and Phase 7.0 versions of the Angiovue software, respectively. Both are calculated from an average value of reflected OCT scan signals that exceed the background noise. However, the new SQI also takes motion artefacts and image defocus into consideration. Numerous previous studies have reported on the OCTA 
image quality using the SSI but more recent studies are using SQI. ${ }^{19,20}$ Direct comparison between these values for individual patient is not possible since users can no longer access the SSI values once the software is upgraded to Phase 7.0. We had the opportunity to do this comparative analysis because we routinely exported the SSI value for our scans prior to the upgrade. We found some agreement between these two indices with a trend for higher SSI values in images with SQI of 8 or 9 compared to those with SQI of $\leq 5,6$ or 7 . However, these indices are not directly interchangeable due to the significant overlap (Table 4).

\section{3 | Optimum thresholds in automated indices for acceptable images}

The minimum cut-off SSI for acceptable OCTA image ranges from 40 to 60 in the current literature. For example, SSI $\geq 40$ was used as the cut-off threshold in the Tang et $\mathrm{al}^{21}$ study for evaluation of quantitative metrics in diabetic patients and the Cao et $\mathrm{al}^{22}$ study evaluating patients with preclinical DR. SSI $\geq 50$ was applied in the studies reported by Samara et $\mathrm{al}^{23}$ Wang et $\mathrm{al}^{24}$ and Seknazi et $\mathrm{al}^{25}$ for quantitative analysis and in the study by Iida et $\mathrm{al}^{3}$ for qualitative assessment of OCTA features in patients with RVO. This threshold was also used in the Hwang et $\mathrm{al}^{26}$ study for quantification of capillary nonperfusion in DR. A higher threshold of SSI $\geq 60$ was used by other studies, such as Mastropasqua et $\mathrm{al}^{27}$ to analyse VR in patients with RVO complicated with macular oedema, and Golebiewska et $\mathrm{al}^{28}$ to assess OCTA features in children with type $1 \mathrm{DM}$. Conversely, in more recent studies using Optovue device, an SQI of $\geq 6$ was used as the threshold for acceptable images. ${ }^{19,20}$ However, none of the above studies provided a scientific justification for the minimum SSI or SQI threshold chosen.

A few studies have investigated which signal strength threshold should be used for excluding OCTA images from quantitative assessment. Fenner et $\mathrm{al}^{29}$ used Topcon DRI OCT Triton imaging system to study how SSI influence the repeatability of VD measurement. These authors reported that SSI $\geq 60$ (Topcon SSI range: 0-100, manufacturer recommended cut-off value: 45 , see Table S4) is required but additional consideration should be given to visibility of the fine vasculature of retinal capillary plexus and motion artefact. This is in agreement with our data showing that SSI alone is not a sufficient indicator for image quality. Therefore, if SSI is the only parameter used for OCTA image quality assessment, additional manual grading is essential to identify image artefacts that may impact on the quantitative analysis. Lim et $\mathrm{al}^{10}$ used the Angioplex from the Zeiss Cirrus 5000 to investigate the effect of signal strength on the
VD, perfusion density (PD) and FAZ. They found that a correlation between VD and PD with increasing signal strength from 7 to 10 (Zeiss signal strength range: 0-10, manufacturer recommended cut-off value: 6 (see Table S4). However, there was no significant difference between signal strengths of 9 and 10 in all quantitative measures of retinal vasculature. Therefore, signal strength of at least 9 instead of 6 was recommended. The optimum thresholds for Optovue-derived SSI and SQI have not been investigated previously.

To determine the validity of thresholds for discarding unacceptable OCTA images acquired on the Optovue device, we created a simple four-level OCTA image quality grading system. Those in grades 1 and 2 were considered unacceptable for quantitative and clinical evaluation due to obvious vessel architecture discontinuity or loss and motion artefacts. We found from the ROC curve that an SSI boundary between 69 and 70 is the optimum cutoff threshold between acceptable (SSI $\geq 70$ ) and unacceptable (SSI $\leq 69)$ images. This is much higher than the manufacturer recommended threshold of $>39$ for acceptable quality. In contrast, an SQI boundary between 6 and 7 was shown as the optimum cut-off threshold between the acceptable (SQI $\geq 7$ ) and unacceptable (SQI $\leq 6)$ image with maximum sensitivity and specificity, and this is a step level higher than the manufacture recommended threshold of $\geq 6$ for acceptable quality. Our results suggest that SQI, the more recently developed automated image quality index, correlates more closely with human assessment and caution should be taken when using SSI as an indicator for image quality assessment. It is important to note that a high cut-off threshold of SQI $\geq 9$ would exclude $74 \%$ of the images that are considered as acceptable; while still allowing 3\% of unacceptable images to be included. Conversely, among the images meeting the recommended SQI $\geq 7$ requirement, $32 \%$ will still be considered as unacceptable based on gold standard manual grading.

\section{4 | Factors affecting OCTA image quality}

Various factors have been shown to affect the scan quality in SD-OCT such as older age, poor vision, high myopia and astigmatism, cortical and post-sub-capsular cataract $^{30,31}$ and other eye pathology. ${ }^{32,33}$ Say et $\mathrm{al}^{34}$ evaluated image quality and the frequency of artefacts in both eyes of patients with unilateral choroidal melanoma using the $3 \mathrm{~mm}$ protocol on Optovue OCTA. They found that diseased eyes, poor visual acuity and male sex were associated with more image artefacts. In agreement with other studies, we found more frequent unacceptable images and low automated quality indices in the diseased 
cohort particularly in eyes with macular atrophy. Therefore, clinical trials of retinal vascular disease that examine OCTA parameters as secondary endpoints need to anticipate high rates $(60 \%-70 \%)$ of failure to acquire acceptable image for quantitative analysis. A previous study suggested that poor visual acuity and unstable fixation contribute to motion artefact and segmentation error. ${ }^{17}$ We showed that among patient factors (age and sex) and ocular factors (visual acuity, eye pathology and macular status) decreased visual acuity was the only significant predictor for unacceptable OCTA image quality.

\section{5 | Limitations}

There are few limitations in our study. First, a portion of the images graded was taken from a retrospective cohort of subjects who had routine clinical imaging. We had only limited range of retinal vascular diseases and the imaging was performed by ophthalmic technicians with varying experience. Therefore, further evaluation is required in a larger and more diverse cohort of patients including patients with macular degeneration. The use of trial certified imaging technician may also reduce the rate of unacceptable images in patient cohort. Second, we only examined and categorized the OCTA images based on the en face superficial capillary plexus. The clarity of vascular tree is influenced by segmentation accuracy in addition to motion artefacts. It is commonly noted that segmentation error is more frequent at the outer retinal layers (retinal pigment epithelium and Bruch's membrane) in macular degeneration. Hence, manual grading of the en face superficial capillary plexus may not be applicable to deep avascular layer OCTA image in patients with choroidal neovascularisation. Nevertheless, our manual grading of superficial capillary plexus is more relevant to the disease cohort that we have chosen to study: DR, vein occlusion and Coats disease. Third, we did not measure the intra individual variation of our manual grading and we did not investigate its impact on the quantitative OCTA measurements and their repeatability. Fourth, the impact of media opacity such as degree of age-related cataract was not taken into account in logistic regression. Finally, our grading system was only validated images from healthy subjects and patients with retinal vascular diseases. It cannot be assumed that the minimum threshold we have recommended is applicable to images from patients with outer retinal vascular pathology such as neovascular AMD. There may be differences in the rate of segmentation error at the irregular retinal pigment epithelium layer commonly seen in AMD compared to the boundaries of the superficial vascular plexus. Further validation study is required in choroidal neovascular diseases.

\section{6 | Conclusions}

We established a simple, easy to learn, manual grading system of the superficial capillary plexus OCTA images. Using this system as a quality measure, we showed that SQI is superior to SSI in discriminating between acceptable and unacceptable images. Our results provide some evidence for using a higher index than manufacturer's recommended minimum threshold for SQI ( $\geq 7$ as compared to $\geq 6$ ) in the current Phase 7.0 software might be preferable. However, caution is required in using these thresholds since up to $30 \%$ of the images with image quality indicators reaching the recommended minimum thresholds were still be considered unacceptable based on manual grading. The high rates of unacceptable OCTA images in patients with retinal vascular disease may limit the utility of OCTA-derived vascular metrics as a clinical trials endpoint measure.

\section{FINANCIAL DISCLOSURE}

This study was supported by grants from National Health and Medical Research Council Career Development Fellowship (APP1142962, F.K.C.), Centre of Research Excellence (APP1116360, F.K.C.) and the Egyptian Ministry of Higher Education (N.A.).

\section{CONFLICT OF INTEREST}

None declared.

\section{ORCID}

Fred K. Chen (1) https://orcid.org/0000-0003-2809-9930

\section{REFERENCES}

1. Sambhav K, Grover S, Chalam KV. The application of optical coherence tomography angiography in retinal diseases. Surv Ophthalmol. 2017;62(6):838-866.

2. Coscas G, Lupidi M, Coscas F. Optical coherence tomography angiography in diabetic maculopathy. Dev Ophthalmol. 2017; 60:38-49.

3. Iida $Y$, Muraoka $Y$, Ooto S, et al. Morphologic and functional retinal vessel changes in branch retinal vein occlusion: an optical coherence tomography angiography study. Am J Ophthalmol. 2017;182:168-179.

4. Battaglia Parodi M, Romano F, Cicinelli MV, et al. Retinal vascular impairment in best vitelliform macular dystrophy assessed by means of optical coherence tomography angiography. Am J Ophthalmol. 2018;187:61-70.

5. Ahmed D, Stattin M, Graf A, et al. Detection of treatmentnaive choroidal neovascularization in age-related macular degeneration by swept source optical coherence tomography angiography. Retina. 2018;38(11):2143-2149.

6. Sellam A, Glacet-Bernard A, Coscas F, Miere A, Coscas G, Souied EH. Qualitative and quantitative follow-up using optical coherence tomography angiography of retinal vein occlusion 
treated with anti-VEGF: optical coherence tomography angiography follow-up of retinal vein occlusion. Retina. 2017;37(6): 1176-1184.

7. Toto L, D'Aloisio R, Di Nicola M, et al. Qualitative and quantitative assessment of vascular changes in diabetic macular edema after dexamethasone implant using optical coherence tomography angiography. Int J Mol Sci. 2017;18(6):1181.

8. Tan ACS, Tan GS, Denniston AK, et al. An overview of the clinical applications of optical coherence tomography angiography. Eye (Lond). 2018;32(2):262-286.

9. Venugopal JP, Rao HL, Weinreb RN, et al. Repeatability of vessel density measurements of optical coherence tomography angiography in normal and glaucoma eyes. $\mathrm{Br} J$ Ophthalmol. 2018;102(3):352-357.

10. Lim HB, Kim YW, Kim JM, Jo YJ, Kim JY. The importance of signal strength in quantitative assessment of retinal vessel density using optical coherence tomography angiography. Sci Rep. 2018;8(1):12897.

11. Zhang X, Iverson SM, Tan O, Huang D. Effect of signal intensity on measurement of ganglion cell complex and retinal nerve fiber layer scans in fourier-domain optical coherence tomography. Transl Vis Sci Technol. 2015;4(5):7.

12. Al-Sheikh M, Ghasemi Falavarjani K, Akil H, Sadda SR. Impact of image quality on OCT angiography based quantitative measurements. Int J Ret Vitr. 2017;3:13.

13. Scanlon $\mathrm{PH}$. The English national screening programme for sight-threatening diabetic retinopathy. J Med Screen. 2008;15 (1):1-4.

14. Kraus MF, Potsaid B, Mayer MA, et al. Motion correction in optical coherence tomography volumes on a per A-scan basis using orthogonal scan patterns. Biomed Opt Express. 2012;3(6): 1182-1199.

15. Jia Y, Tan O, Tokayer J, et al. Split-spectrum amplitudedecorrelation angiography with optical coherence tomography. Opt Express. 2012;20(4):4710-4725.

16. Munk MR, Giannakaki-Zimmermann H, Berger L, et al. OCTangiography: a qualitative and quantitative comparison of 4 OCT-A devices. PLoS One. 2017;12(5):e0177059.

17. Lauermann JL, Woetzel AK, Treder M, et al. Prevalences of segmentation errors and motion artifacts in oct-angiography differ among retinal diseases. Graefes Arch Clin Exp Ophthalmol. 2018;256(10):1807-1816.

18. Nobre Cardoso J, Keane PA, Sim DA, et al. Systematic evaluation of optical coherence tomography angiography in retinal vein occlusion. Am J Ophthalmol. 2016;163:93-107.e106.

19. Schwartz R, Sivaprasad S, Macphee R, et al. Subclinical macular changes and disease laterality in pediatric coats disease determined by quantitative optical coherence tomography angiography. Retina. 2018. https://doi.org/10.1097/IAE. 0000000000002322

20. Liu L, Gao J, Bao W, et al. Analysis of foveal microvascular abnormalities in diabetic retinopathy using optical coherence tomography angiography with projection artifact removal. J Ophthalmol. 2018;2018:1-9.

21. Tang FY, Ng DS, Lam A, et al. Determinants of quantitative optical coherence tomography angiography metrics in patients with diabetes. Sci Rep. 2017;7(1):2575.

22. Cao D, Yang D, Huang Z, et al. Optical coherence tomography angiography discerns preclinical diabetic retinopathy in eyes of patients with type 2 diabetes without clinical diabetic retinopathy. Acta Diabetol. 2018;55(5):469-477.

23. Samara WA, Shahlaee A, Sridhar J, Khan MA, Ho AC, Hsu J. Quantitative optical coherence tomography angiography features and visual function in eyes with branch retinal vein occlusion. Am J Ophthalmol. 2016;166:76-83.

24. Wang Q, Chan SY, Yan Y, et al. Optical coherence tomography angiography in retinal vein occlusions. Graefes Arch Clin Exp Ophthalmol. 2018;256(9):1615-1622.

25. Seknazi D, Coscas F, Sellam A, et al. Optical coherence tomography angiography in retinal vein occlusion: correlations between macular vascular density, visual acuity, and peripheral nonperfusion area on fluorescein angiography. Retina (Philadelphia, PA). 2018;38(8):1562-1570.

26. Hwang TS, Gao SS, Liu L, et al. Automated quantification of capillary nonperfusion using optical coherence tomography angiography in diabetic retinopathy. JAMA Ophthalmol. 2016; 134(4):367-373.

27. Mastropasqua R, Toto L, Di Antonio L, et al. Optical coherence tomography angiography microvascular findings in macular edema due to central and branch retinal vein occlusions. Sci Rep. 2017;7:40763.

28. Golebiewska J, Olechowski A, Wysocka-Mincewicz M, et al. Optical coherence tomography angiography vessel density in children with type 1 diabetes. PLoS One. 2017;12(10):e0186479.

29. Fenner BJ, Tan GSW, Tan ACS, Yeo IYS, Wong TY, Cheung GCM. Identification of imaging features that determine quality and repeatability of retinal capillary plexus density measurements in oct angiography. Br J Ophthalmol. 2018;102(4):509-514.

30. Lee R, Tham YC, Cheung CY, et al. Factors affecting signal strength in spectral-domain optical coherence tomography. Acta Ophthalmol. 2018;96(1):e54-e58.

31. Na JH, Sung KR, Lee Y. Factors associated with the signal strengths obtained by spectral domain optical coherence tomography. Korean J Ophthalmol. 2012;26(3):169-173.

32. Asrani S, Essaid L, Alder BD, Santiago-Turla C. Artifacts in spectral-domain optical coherence tomography measurements in glaucoma. JAMA Ophthalmol. 2014;132(4):396-402.

33. Song Y, Lee BR, Shin YW, Lee YJ. Overcoming segmentation errors in measurements of macular thickness made by spectraldomain optical coherence tomography. Retina. 2012;32(3):569-580.

34. Say EAT, Ferenczy S, Magrath GN, Samara WA, Khoo CTL, Shields CL. Image quality and artifacts on optical coherence tomography angiography: comparison of pathologic and paired fellow eyes in 65 patients with unilateral choroidal melanoma treated with plaque radiotherapy. Retina. 2017;37(9):1660-1673.

\section{SUPPORTING INFORMATION}

Additional supporting information may be found online in the Supporting Information section at the end of this article.

How to cite this article: Ali N, Sampson DM, Yong AA, et al. Clinical validation of the RTVue optical coherence tomography angiography image quality indicators. Clin Experiment Ophthalmol. 2019;1-12. https://doi.org/10.1111/ceo.13680 